\title{
Combined Interventions for Severe Novel Coronavirus Disease (COVID-19): Experience from 350 Patients
}

This article was published in the following Dove Press journal: Infection and Drug Resistance

Ting Guo, ${ }^{1-3, *}$ Qinxue Shen, ${ }^{1-3, *}$ Zhiguo Zhou, ${ }^{4}$ Jinhua $\mathrm{Li}^{\mathrm{I}}{ }^{\mathrm{I}}{ }^{-3}$ Wei Guo, ${ }^{1-3}$ Wenlong He, ${ }^{1-3}$ Yunnian Wang, ${ }^{5}$ Zhi Xiang, ${ }^{6}$ Peng Huang, ${ }^{7}$ Nanyang Zeng, ${ }^{8}$ Qingwu Qin, ${ }^{1-3}$ Ping Chen, ${ }^{1-3}$ Hong Luo, (D) ${ }^{1-3}$ Hong Peng $\left(\mathbb{D}^{1-3}\right.$

'Department of Respiratory and Critical Care Medicine, The Second Xiangya Hospital of Central-South University, Changsha, Hunan, People's Republic of China; ${ }^{2}$ Research Unit of Respiratory Disease, Central-South University, Changsha, Hunan, People's Republic of China; ${ }^{3}$ The Respiratory Disease Diagnosis and Treatment Center of Hunan Province, Changsha, Hunan, People's Republic of China; ${ }^{4}$ Department of Respiratory and Critical Care Medicine, The First Hospital of Changsha, Changsha, Hunan, People's Republic of China; ${ }^{5}$ Department of Respiratory and Critical Care Medicine, The Traditional Chinese Medicine Hospital of Liling, Zhuzhou, Hunan, People's Republic of China; ${ }^{6}$ Department of Respiratory and Critical Care Medicine, The First People's Hospital of Huaihua, Huaihua, Hunan, People's Republic of China; ${ }^{7}$ Department of Respiratory and Critical Care Medicine, The Central Hospital of Zhuzhou, Zhuzhou, Hunan, People's Republic of China; ${ }^{8}$ Department of Infectious Disease, The Lvkou People's Hospital of Zhuzhou, Zhuzhou, Hunan, People's Republic of China

*These authors contributed equally to this work

Correspondence: Hong Peng Department of Respiratory and Critical Care Medicine, The Second Xiangya Hospital of Central-South University, No. 139 Renmin Middle Road, Changsha, Hunan 4I00I I, People's Republic of China Tel +86 I3807494486

Email penghong66@csu.edu.cn
Purpose: To summarize the clinical features and effective therapy of severe COVID-19 patients.

Patients and Methods: In this retrospective, multicenter study, the medical records of COVID-19 patients in Hunan, from January 21, 2020 to February 19, 2020 were reviewed. Results: Of the 350 COVID-19 patients, $13.7 \%$ were severe cases. On admission, compared with non-severe patients, more severe patients had a neutrophil/lymphocyte ratio $>3(58.3 \% \mathrm{vs}$ $33.8 \%, P=0.001)$, D-dimer $>1 \mathrm{mg} / \mathrm{L}(41.7 \%$ vs $13.6 \%, P<0.0001)$, higher level of CRP (39.1 mg/L, IQR18.1-75.9 vs $13.4 \mathrm{mg} / \mathrm{L}$, IQR5.0-32.8, $P<0.0001$ ), and multiple pneumonia on CT $(77.1 \%$ vs $18.2 \%, P<0.0001)$. All severe patients received oxygen support. $95.8 \%$ of them received antivirals, and the most frequent therapy was lopinavir and ritonavir plus human interferon- $\alpha 2 \mathrm{~b}$. Moxifloxacin was used in $70.8 \%$ severe patients. The total dosage of methylprednisolone sodium succinate was $640 \mathrm{mg}$ (IQR 360-960) in severe patients, and the duration of use was 8.5 days (IQR 6.8-11.3). The total dosage of immunoglobulin was $80 \mathrm{~g}$ (IQR, 60-140) in severe patients, and the duration was 8.0 days (IQR, 6.0-11.5). As of March 15, $2020,95.8 \%$ of the severe patients had been discharged and only two deaths occurred.

Conclusion: The rate of severe cases and mortality of COVID-19 in Hunan are lower than those in Wuhan. In addition to antivirals and oxygen support, timely interventions including corticosteroids, immunoglobulin, and antibiotics, contribute to improving the prognosis of severe COVID-19 patients.

Keywords: coronavirus disease 2019, SARS-CoV-2, severe disease, corticosteroid, immunoglobulin

\section{Introduction}

Severe acute respiratory syndrome coronavirus 2 (SARS-CoV-2) is a novel type of beta coronavirus, ${ }^{1}$ and has resulted in the large-scale transmission of 2019 novel coronavirus disease (COVID-19) since December 2019 from Wuhan, Hubei Province, China. Thus far, in mainland China, over eighty thousand people have been infected with SARS-CoV-2, and more than three thousand patients have died of COVID-19. ${ }^{2}$ More seriously, an increasing number of cases have been confirmed in other countries and continents, especially in countries such as United States of America, Brazil, India, Russia, and South Africa, the number of COVID-19 patients has increased rapidly. ${ }^{3}$ The World Health Organization (WHO) classified the COVID-19 outbreak as a global pandemic on March 12, 2020. ${ }^{4}$ COVID-19 has drawn intensive attention and poses a threat to global public health. 
According to the first clinical report of the 41 earliest COVID-19 cases in Wuhan, 32\% of patients were admitted to the intensive care unit (ICU) and half of them died. ${ }^{5}$ Other studies in Wuhan also reported high incidences of severe disease, with rates of $23-41.4 \% .^{6-8}$ With the transmission of SARS-CoV-2 outside of Wuhan, China, the incidence of severe cases has decreased. In Beijing, from January 20 to February 10, 262 patients were confirmed to have SARSCoV-2 infections, and $17.6 \%$ were severe cases. ${ }^{9}$ Other regions in China, such as Zhejiang and Jiangsu, also showed much fewer severe cases of SARS-CoV-2 infection. ${ }^{10,11}$ The decreased incidence of severe cases indicates effective control and treatment of the SARS-CoV-2 infection in China. As COVID-19 is rapidly spreading in other regions, more information about existing treatment experience especially effective interventions for severe patients, is important due to the high mortality rate.

\section{Materials and Methods}

\section{Study Design and Participants}

In this study, we retrospectively analyzed data from 350 patients with SARS-CoV-2 infections from three different cities in Hunan Province, China, which is a neighbor of Hubei Province, that reported the first COVID-19 patient on January 21,2020 . We aimed to analyze the clinical characteristics and therapeutic features of severe COVID19 patients, to provide information on the effective treatment of severe COVID-19 and reduce the number of poor outcomes.

Patients from three different cities in Hunan Province with laboratory-confirmed SARS-CoV-2 infections according to the WHO interim guidelines were enrolled in this study. The three designated hospitals in these cities for COVID-19 treatment included the Public Health Clinic Center of Changsha City, Central Hospital of Zhuzhou City, and First People's Hospital of Huaihua City. The first COVID-19 case in Hunan Province was reported on January 21, 2020, in Changsha. The medical records of COVID-19 patients who were referred to these hospitals from January 21, 2020, to February 19, 2020, were retrospectively reviewed and analyzed. The clinical outcomes were followed up to March 15, 2020. The features were compared between severe and non-severe COVID-19 cases. The risk factors for severe illness were assessed.

This study was approved by the Medical Ethical Committees of the Public Health Clinic Center of Changsha City, Central Hospital of Zhuzhou City, First
People's Hospital of Huaihua City, and the Second Xiangya Hospital of Central South University (Approved Number. KL-2,020,020), and the requirement for informed consent was waived based on the CIOMS guidelines.

\section{Definitions}

Based on the New Coronavirus Pneumonia Prevention and Control Program (Version Six) from the National Health Commission of China, ${ }^{12}$ COVID-19 patients were divided into asymptomatic carriers, mild patients, moderate patients, severe patients, and critically severe patients. Asymptomatic carriers were defined as patients with laboratory-confirmed SARS-CoV-2 infections without symptoms and imaging findings. According to the guidelines from the National Health Commission of China, asymptomatic carriers were not classified as confirmed cases. Mild COVID-19 was defined as mild clinical symptoms without imaging findings of pneumonia. Moderate COVID-19 was defined as fever and respiratory symptoms with imaging findings of pneumonia. Severe COVID-19 was defined as any one of the following criteria: 1) respiratory distress with a respiratory rate $\geq 30$ breaths $/ \mathrm{min} ; 2$ ) pulse oximeter oxygen Saturation $\left(\mathrm{SpO}_{2}\right) \leq 93 \%$ in the resting-state; 3) $\mathrm{PaO} 2 / \mathrm{FiO} 2 \leq 300 \mathrm{mmHg}(1 \mathrm{mmHg}=0.133$ $\mathrm{kPa})$. And critically severe COVID-19 was defined as any one of the following criteria: 1) respiratory failure in need of mechanical ventilation; 2) shock; 3) with other organ dysfunction. In our study, mild patients and moderate patients were included in the non-severe group, and severe patients as well as critically severe patients were included in the severe group.

The patients with a history of exposure to Wuhan were defined as those with prior tourism or residence in Wuhan within 14 days before illness onset.

\section{Data Collection}

Epidemiological information, clinical manifestations, laboratory and radiological results, treatment, and outcome data were obtained from the medical records. Any missing or uncertain records were collected and clarified through direct communications with the patients or their families. All data were double-checked by two independent physicians (Jinhua Li and Wei Guo).

\section{Statistical Analysis}

Statistical analyses were performed with SPSS (version 26.0). Continuous variables are described as the median (interquartile range, IQR). Categorical variables are 
presented as counts (\%). The medians (IQRs) were compared with the Mann-Whitney $U$-test. Categorical variables were compared with the $\chi^{2}$ test or Fisher's exact test. To explore the risk factors associated with severe illness, univariable and multivariable logistic regression models were used. $P<0.05$ was considered statistically significant.

\section{Results}

\section{Epidemiological and Baseline Characteristics}

By February 19, 2020, a total of 373 admitted hospital patients were identified as having laboratory-confirmed SARS-CoV-2 infections in Changsha city, Zhuzhou city, and Huaihua city. Twenty-three patients were asymptomatic carriers. The baseline data of the 350 confirmed patients enrolled are shown in Table 1. Forty-eight (13.7\%) were severe patients, and $302(86.3 \%)$ were non-severe cases. In the severe group, there were $15(4.3 \%)$ critically severe patients. The clinical types of all patients are shown in Figure 1. The median age of the severe patients was older than that of the non-severe patients (61 years, IQR 46-67 years vs 42 years, IQR $32-52$ years; $P<0.0001)$. None of the severe patients were aged under 14 years, $6(12.5 \%)$ were aged $15-29$ years, $18(37.5 \%)$ were aged $30-59$ years, and $24(50 \%)$ were aged 60 years and older. The age distributions of non-severe patients were different from those of severe patients $[P<0.0001]$, as $13(4.3 \%)$ were aged under 14 years, 47 (15.6\%) were aged 15-29 years, $189(62.6 \%)$ were aged $30-59$ years, $53(17.5 \%)$ were aged 60 years and older. The age distributions of all patients were shown in Figure 2. There were no differences regarding sex, history of exposure to Wuhan, or family cluster cases between the two groups. Approximately $25.0 \%$ of severe patients had a history of smoking, which was different from the nonsevere group (6.3\%) $[P=0.0002]$. There were more comorbidities in severe patients $[P<0.0001]$, as $29(60.4 \%)$ of the severe patients had underlying disease, including hypertension $(16,33.3 \%)$, diabetes $(9,18.8 \%)$, chronic liver disease $(5,10.4 \%)$, chronic kidney disease $(2,4.2 \%)$, cardiovascular disease $(8,16.7 \%)$, cerebral infarction $(3,6.3 \%)$, and chronic pulmonary disease $(6,12.5 \%)$.

On admission, fever (43, 89.6\%), cough (32, 66.7\%), dyspnea $(28,58.3 \%)$, and fatigue $(17,35.4 \%)$ were the most frequent symptoms in severe patients. There were more severe patients with fever than non-severe patients (89.6\% vs $68.2 \%, P=0.0002)$. Severe patients had higher temperatures, as $68.8 \%$ of them had a highest temperature above $38^{\circ} \mathrm{C}$, while the corresponding proportion was $28.4 \%$ in the non-severe group $(P<0.0001)$. Dyspnea and fatigue were also more common in the severe group than in the non-severe group ( $35.4 \%$ vs $15.9 \%, P=0.001 ; 58.3 \%$ vs $7.3 \%, P<0.0001)$. Among the 222 patients who could provide the exact time of close contact with someone confirmed or suspected to have a SARS-CoV-2 infection, the median incubation period from exposure to symptoms onset in severe patients was 5 days (IOR, 4-10 days). There was no difference in the incubation period between the two groups $[P=0.808]$. There was no obvious difference regarding the median time from illness onset to hospital admission between severe and non-severe patients, and this duration was 5 days (IOR, 3-7 days) in the severe group and 5 days (IOR, $3-8$ days) in the nonsevere group $[P=0.758]$.

\section{Laboratory and Imaging Features}

On admission, the median neutrophil count was $3.9 \times 10^{9} / \mathrm{L}$ (IQR, 2.8-6.1) in severe patients and $2.9 \times 10^{9} / \mathrm{L}$ (IQR, 2.1-3.8) in non-severe patients, which showed a statistically significant $P$ value $<0.0001$ (Table 2). Lymphocytes were below the normal range in 18 (37.5\%) severe patients. The severe patients showed more lymphopenia than non-severe patients $(21.5 \%)$ $[P=0.016]$. More severe patients had neutrophil/lymphocyte ratio $>3$ than non-severe patients $(58.3 \%$ vs $33.8 \%$, $P=0.001$ ), and more severe patients showed thrombopenia than non-severe patients $(16.7 \%$ vs $4.6 \%, P=0.001)$. D-dimer was above $1 \mathrm{mg} / \mathrm{L}$ in $41.7 \%$ of severe patients, which was significantly different from the corresponding proportion of non-severe group (13.6\%) $[P<0.0001]$. More severe patients had a prolonged activated partial thromboplastin time (APTT) than non-severe patients $(8.3 \%$ vs $2.3 \%, P=0.026)$. The median albumin level was lower in severe patients than in the non-severe group (median 35.8 $\mathrm{g} / \mathrm{L}, \mathrm{IQR}, 31.8-39.5$ vs median $38.2 \mathrm{~g} / \mathrm{L}, \mathrm{IQR}, 35.3-41.4$ ) $[P=0.001]$. The aspartate aminotransferase (AST) level was increased in $29.2 \%$ of severe patients, and this proportion was lower in the non-severe group (11.9\%) [ $P=0.002]$. Elevated lactate dehydrogenase (LDH) was more common in severe patients than in non-severe patients $(50 \%$ vs $23.2 \%, P<0.0001)$. More severe patients $(22.9 \%)$ had elevated blood urea nitrogen (BUN) than non-severe patients (3.3\%) $[P<0.0001]$. Severe patients showed a higher median level of creatine kinase (CK) than non-severe patients (median $101 \mathrm{U} / \mathrm{L}, \mathrm{IQR}, 174-956$ vs median 68 U/L, IQR, 
Table I Demographics and Baseline Characteristics of Patients with SARS-CoV-2 Infection

\begin{tabular}{|c|c|c|c|c|}
\hline & $\begin{array}{l}\text { All Patients } \\
(n=350)\end{array}$ & Severe Patients $(n=48)$ & $\begin{array}{l}\text { Non-Severe Patients } \\
(n=302)\end{array}$ & $P$ value \\
\hline \multicolumn{5}{|l|}{ Characteristics } \\
\hline Age, (years) & $43(32-56)$ & $6 I(46-67)$ & $42(32-52)$ & $<0.0001$ \\
\hline \multicolumn{5}{|l|}{ Age groups } \\
\hline$\leq 14$ & $13(3.7)$ & 0 & $13(4.3)$ & $<0.0001$ \\
\hline $15-29$ & $53(15.1)$ & $6(12.5)$ & $47(15.6)$ & \\
\hline $30-59$ & $207(59.1)$ & $18(37.5)$ & $189(62.6)$ & \\
\hline$\geq 60$ & $77(22.0)$ & $24(50.0)$ & $53(17.5)$ & \\
\hline \multicolumn{5}{|l|}{ Sex } \\
\hline Male & 173(49.4) & $29(60.4)$ & |44(47.6) & 0.092 \\
\hline Female & $177(50.6)$ & $19(39.6)$ & $158(52.3)$ & \\
\hline Wuhan exposure & 137(39.1) & $21(43.8)$ & $116(38.4)$ & $0.48 I$ \\
\hline Family cluster case & $166(47.4)$ & $22(45.8)$ & |44(47.7) & 0.243 \\
\hline Smoking & $3 \mathrm{I}(8.9)$ & $12(25.0)$ & $19(6.3)$ & 0.0002 \\
\hline Chronic medical illness & $108(30.9)$ & $29(60.4)$ & $79(26.2)$ & $<0.0001$ \\
\hline Hypertension & $5 I(14.6)$ & $16(33.3)$ & $35(I I .6)$ & 0.0003 \\
\hline Diabetes & $26(7.4)$ & $9(18.8)$ & $17(5.6)$ & 0.001 \\
\hline Cardiovascular disease & $15(4.3)$ & $8(16.7)$ & $7(2.3)$ & $<0.0001$ \\
\hline Chronic Pulmonary disease & $7(2.0)$ & $6(12.5)$ & $\mathrm{I}(0.3)$ & $<0.0001$ \\
\hline Chronic kidney disease & $9(2.6)$ & $2(4.2)$ & $7(2.3)$ & 0.455 \\
\hline Chronic liver disease & |4(4.0) & $5(10.4)$ & $9(3.0)$ & 0.015 \\
\hline Cerebral infarction & $8(2.3)$ & $3(6.3)$ & $5(I .7)$ & 0.049 \\
\hline Malignancy & $\mathrm{I}(0.3)$ & $0(0.0)$ & $\mathrm{I}(0.3)$ & 0.689 \\
\hline \multicolumn{5}{|l|}{ Signs and symptoms } \\
\hline Fever & 249(7I.I) & $43(89.6)$ & $206(68.2)$ & 0.0002 \\
\hline Highest Temperature $\left({ }^{\circ} \mathrm{C}\right)$ & $38.0(37.2-38.4)$ & $38.6(38.0-38.9)$ & $37.8(37.2-37.8)$ & $<0.0001$ \\
\hline$<37.3$ & $101(28.9)$ & $5(10.4)$ & $96(31.8)$ & $<0.0001$ \\
\hline $37.3-38.0$ & $130(37.1)$ & $10(20.8)$ & $120(39.7)$ & \\
\hline $38.1-39.0$ & $97(27.7)$ & $25(52.1)$ & $72(23.8)$ & \\
\hline$>39.0$ & $22(6.3)$ & $8(16.7)$ & I4(4.6) & \\
\hline Cough & $205(58.6)$ & $32(66.7)$ & $173(57.3)$ & 0.220 \\
\hline Fatigue & $65(18.6)$ & $17(35.4)$ & $48(15.9)$ & 0.001 \\
\hline Myalgia & $19(5.4)$ & $2(4.2)$ & $17(5.6)$ & 0.678 \\
\hline Dyspnea & $50(14.3)$ & $28(58.3)$ & $22(7.3)$ & $<0.0001$ \\
\hline Diarrhea & $24(6.9)$ & $6(12.5)$ & $18(6.0)$ & 0.096 \\
\hline Anorexia & $18(5.1)$ & $4(8.3)$ & $14(4.6)$ & 0.281 \\
\hline Vomiting & $10(2.9)$ & $3(6.3)$ & $7(2.3)$ & 0.129 \\
\hline Headache & $23(6.6)$ & $6(12.5)$ & $17(5.6)$ & 0.074 \\
\hline Days of incubation period & $7(3-10)$ & $5(4-10)$ & $7(3-10)$ & 0.808 \\
\hline Days from illness onset to hospital admission & $5(3-7)$ & $5(3-7)$ & $5(3-8)$ & 0.758 \\
\hline
\end{tabular}

Notes: Data are presented as median, interquartile range or $\mathrm{n}(\%)$.

46-105) $[P=0.004]$. Regarding the infection index, the levels of $\mathrm{C}$-reactive protein (CRP) and procalcitonin (PCT) were increased in $83.3 \%$ and $47.9 \%$ of severe patients, respectively. The median level of CRP in the severe group on admission was $39.1 \mathrm{mg} / \mathrm{L}$ (IQR,
18.1-75.9), which was higher than that in the non-severe group (13.4 mg/L, IQR, 5.0-32.8) [ $P<0.0001]$. On admission, more severe patients $(77.1 \%)$ showed multiple pneumonia lesions on images than non-severe patients (18.2\%) $[P<0.0001]$. Typical images of severe and non-severe 


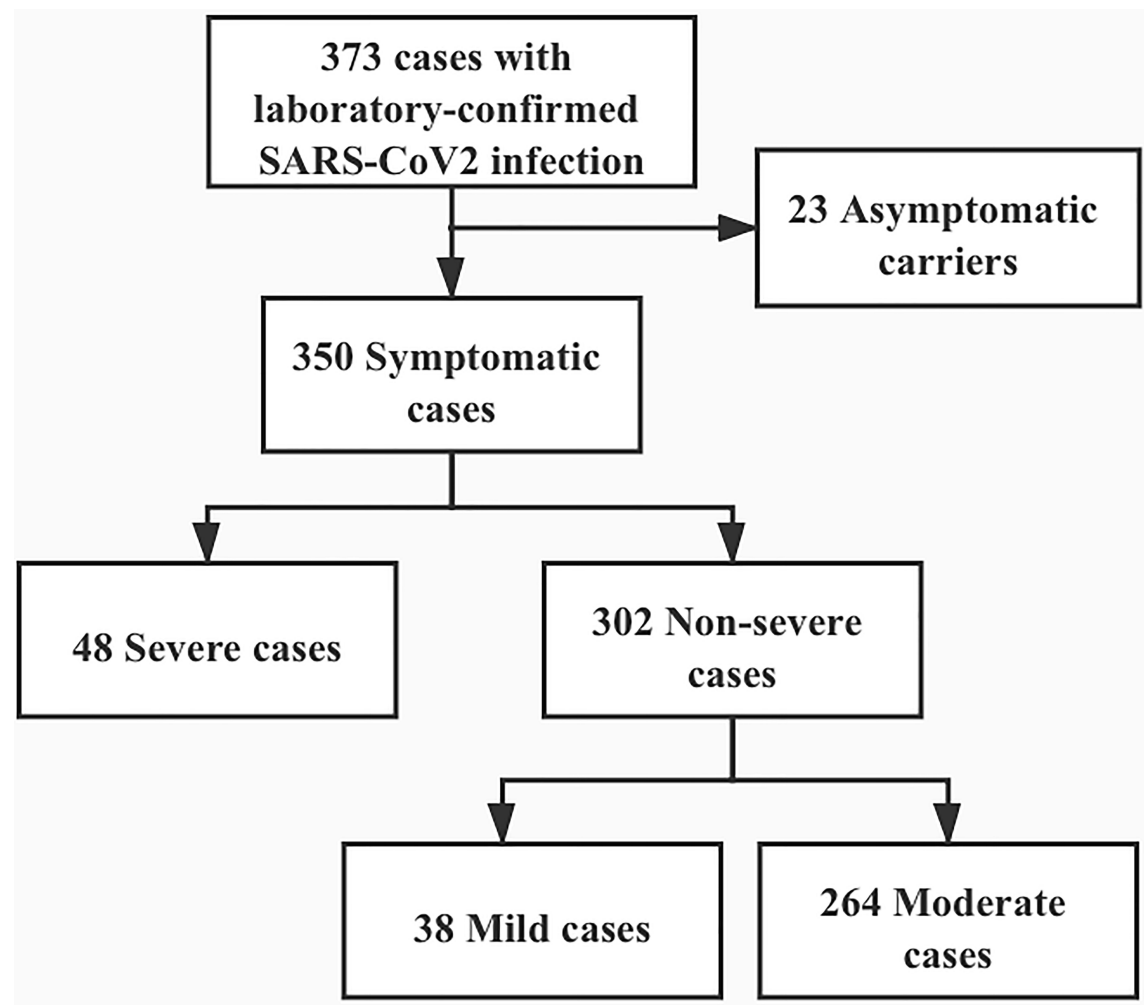

Figure I Flow chart summarizing the enrollment of COVID-19 patients.

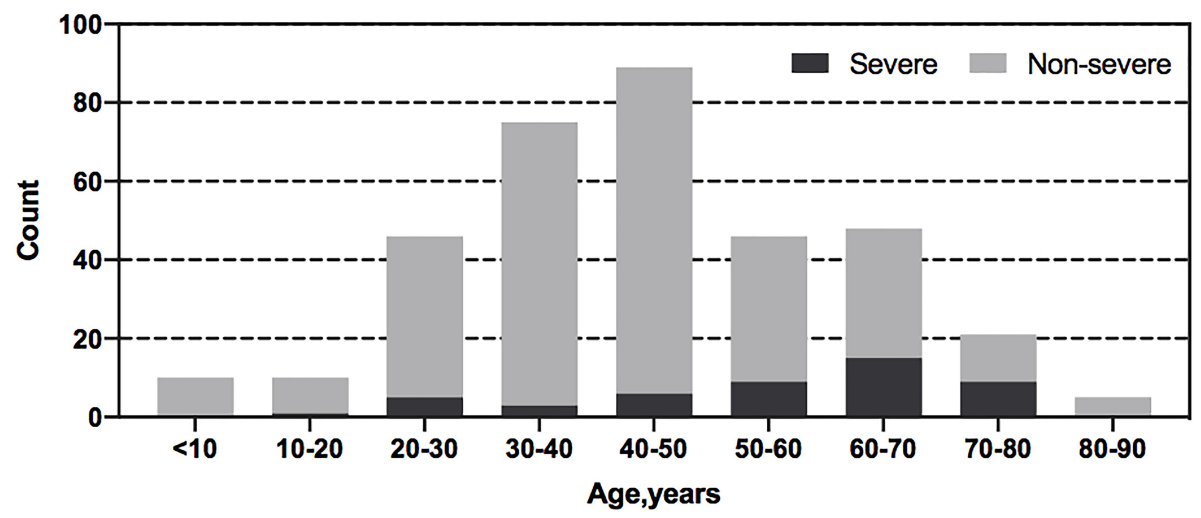

Figure 2 Age distributions of confirmed COVID-19 patients.

patients are shown in Figure 3. 316 patients received at least twice chest $\mathrm{CT}$ examination during hospitalization. In the severe group, 26 patients $(54.2 \%)$ had a resolution of lesions at their second chest CT examination, which was lower than that in 268 non-severe patients who had taken a second chest $\mathrm{CT}$ examination (72.8\%) $[P=0.01]$.

\section{Treatment and Clinical Outcomes}

During hospitalization, $25.0 \%$ of severe patients had complications, including acute respiratory distress syndrome (ARDS) $(8.3 \%)$, acute cardiac injury (4.2\%), acute kidney injury (8.3\%), sepsis (8.3\%), allergic eruption (4.2\%), phlebothrombosis (2.1\%), and pneumothorax (2.1\%) (Table 3). Non-severe patients showed fewer complications $(2.3 \%)$ $[P<0.0001]$. The treatments for the two groups were quite different. No difference was found in the usage of antivirals between the two groups ( $95.8 \%$ vs $92.7 \%, P=0.553)$, and lopinavir and ritonavir per os plus recombinant human interferon- $\alpha 2 \mathrm{~b}$ through aerosol inhalation was used most frequently $(44.9 \%)$. Compared with the number of nonsevere patients, more severe patients received antibiotics (100\% vs $42.7 \%, P<0.0001)$, corticosteroids $(95.8 \%$ vs 
Table 2 Laboratory and Image Findings of Patients Infected with SARS-CoV-2 on Admission to Hospital

\begin{tabular}{|c|c|c|c|c|c|}
\hline & $\begin{array}{l}\text { Normal } \\
\text { Range }\end{array}$ & $\begin{array}{l}\text { All Patients } \\
(n=350)\end{array}$ & $\begin{array}{l}\text { Severe Patients } \\
(n=48)\end{array}$ & $\begin{array}{l}\text { Non-Severe } \\
\text { Patients } \\
(n=302)\end{array}$ & $P$ value \\
\hline $\begin{array}{l}\text { White blood cell count, } \times 10^{9} / \mathrm{L} \\
<4 \\
4-10 \\
>10\end{array}$ & $4-10$ & $\begin{array}{l}4.7(3.6-6.1) \\
126(36.0) \\
206(58.9) \\
18(5.1)\end{array}$ & $\begin{array}{l}5.2(3.8-6.8) \\
13(27.1) \\
30(62.5) \\
5(10.4)\end{array}$ & $\begin{array}{l}4.6(3.6-6.0) \\
113(37.4) \\
176(58.3) \\
13(4.3)\end{array}$ & $\begin{array}{l}0.078 \\
0.113\end{array}$ \\
\hline $\begin{array}{l}\text { Neutrophil count, } \times 10^{9} / \mathrm{L} \\
<2 \\
2-7 \\
>7\end{array}$ & $2-7$ & $\begin{array}{l}3.0(2.2-4.1) \\
70(20.0) \\
256(73.1) \\
24(6.9)\end{array}$ & $\begin{array}{l}3.9(2.8-6.1) \\
6(12.5) \\
33(68.8) \\
9(18.8)\end{array}$ & $\begin{array}{l}2.9(2.1-3.8) \\
64(21.2) \\
223(73.8) \\
15(5.0)\end{array}$ & $\begin{array}{l}<0.0001 \\
0.001\end{array}$ \\
\hline $\begin{array}{l}\text { Lymphocyte count, } \times 10^{9} / \mathrm{L} \\
<0.8\end{array}$ & $0.8-4$ & $\begin{array}{l}1.2(0.9-1.69) \\
83(23.7)\end{array}$ & $\begin{array}{l}1.0(0.7-1.5) \\
18(37.5)\end{array}$ & $\begin{array}{l}1.2(0.9-1.7) \\
65(21.5)\end{array}$ & $\begin{array}{l}0.028 \\
0.016\end{array}$ \\
\hline $\begin{array}{l}\text { Neutrophil/Lymphocyte } \\
\quad<3 \\
>3\end{array}$ & & $\begin{array}{l}2.4(1.6-4.0) \\
220(62.9) \\
130(37.1)\end{array}$ & $\begin{array}{l}3.8(2.3-7.9) \\
20(41.7) \\
28(58.3)\end{array}$ & $\begin{array}{l}2.2(1.5-3.5) \\
200(66.2) \\
102(33.8)\end{array}$ & $\begin{array}{l}<0.0001 \\
0.001\end{array}$ \\
\hline $\begin{array}{l}\text { Hemoglobin, g/L } \\
\quad<110\end{array}$ & $110-160$ & $\begin{array}{l}|3|(\mid 20-142) \\
26(7.4)\end{array}$ & $\begin{array}{l}125(1 \mid 4-142) \\
5(10.4)\end{array}$ & $\begin{array}{l}132(|2|-|4|) \\
21(7.0)\end{array}$ & $\begin{array}{l}0.090 \\
0.395\end{array}$ \\
\hline $\begin{array}{l}\text { Platelet count, } \times 10^{9} / \mathrm{L} \\
\quad<100\end{array}$ & $100-300$ & $\begin{array}{l}183(144-245) \\
22(6.3)\end{array}$ & $\begin{array}{l}171(117-232) \\
8(16.7)\end{array}$ & $\begin{array}{l}285(147-245) \\
14(4.6)\end{array}$ & $\begin{array}{l}0.076 \\
0.001\end{array}$ \\
\hline $\begin{array}{l}\text { PT, s } \\
\qquad>15\end{array}$ & $10-15$ & $\begin{array}{l}12.3(11.3-13.2) \\
36(10.3)\end{array}$ & $\begin{array}{l}12.5(11.2-13.4) \\
6(12.5)\end{array}$ & $\begin{array}{l}12.3(11.4-13.2) \\
30(9.9)\end{array}$ & $\begin{array}{l}0.845 \\
0.587\end{array}$ \\
\hline $\begin{array}{l}\text { APTT, s } \\
\quad>46\end{array}$ & $26.2-46$ & $\begin{array}{l}32.2(29.9-35.5) \\
\mathrm{II}(3.1)\end{array}$ & $\begin{array}{l}22.5(29.1-33.5) \\
4(8.3)\end{array}$ & $\begin{array}{l}32.1(30.2-35.2) \\
7(2.3)\end{array}$ & $\begin{array}{l}0.128 \\
0.026\end{array}$ \\
\hline $\begin{array}{l}\text { D-dimer, mg/L } \\
>>1\end{array}$ & $0-1$ & $\begin{array}{l}0.4(0.2-0.7) \\
61(17.4)\end{array}$ & $\begin{array}{l}0.7(0.3-3.3) \\
20(4 I .7)\end{array}$ & $\begin{array}{l}0.4(0.2-0.7) \\
4 I(13.6)\end{array}$ & $\begin{array}{l}0.001 \\
<0.0001\end{array}$ \\
\hline $\begin{array}{l}\text { Albumin, g/L } \\
\quad<35\end{array}$ & $35-55$ & $\begin{array}{l}37.9(35.4-40.9) \\
80(22.9)\end{array}$ & $\begin{array}{l}35.8(31.8-39.5) \\
21(43.8)\end{array}$ & $\begin{array}{l}38.2(35.3-41.4) \\
59(19.5)\end{array}$ & $\begin{array}{l}0.001 \\
<0.0001\end{array}$ \\
\hline $\begin{array}{l}\text { Globulin, g/L } \\
\quad>29.5 \\
20.2-29.5 \\
<20.2\end{array}$ & $20.2-29.5$ & $\begin{array}{l}25.9(23.8-28.3) \\
64(18.3) \\
272(77.7) \\
14(4.0)\end{array}$ & $\begin{array}{l}26.5(24.5-28.9) \\
10(20.8) \\
37(77.1) \\
1(2.1)\end{array}$ & $\begin{array}{l}25.7(23.5-28.2) \\
54(17.9) \\
235(77.8) \\
13(4.3)\end{array}$ & $\begin{array}{l}0.047 \\
0.701\end{array}$ \\
\hline $\begin{array}{c}\text { ALT, U/L } \\
>42\end{array}$ & $0-42$ & $\begin{array}{l}20.6(15.0-28.0) \\
39(11.1)\end{array}$ & $\begin{array}{l}19.9(14.9-30.4) \\
7(14.6)\end{array}$ & $\begin{array}{l}20.7(15.0-27.8) \\
32(10.6)\end{array}$ & $\begin{array}{l}0.968 \\
0.415\end{array}$ \\
\hline $\begin{array}{c}\text { AST, U/L } \\
>37\end{array}$ & $0-37$ & $\begin{array}{l}24.0(19.2-31.1) \\
50(14.3)\end{array}$ & $\begin{array}{l}30.4(23.3-40.8) \\
14(29.2)\end{array}$ & $\begin{array}{l}23.9(19.0-29.1) \\
36(11.9)\end{array}$ & $\begin{array}{l}0.001 \\
0.002\end{array}$ \\
\hline $\begin{array}{l}\text { CK, U/L } \\
\quad>190 \\
\leq 190\end{array}$ & $10-190$ & $\begin{array}{l}71.1(46.9-109.7) \\
32(9.1) \\
318(90.9)\end{array}$ & $\begin{array}{l}101(174-956) \\
9(18.8) \\
39(81.3)\end{array}$ & $\begin{array}{l}68(46-105) \\
23(7.6) \\
279(92.4)\end{array}$ & $\begin{array}{l}0.004 \\
0.013\end{array}$ \\
\hline $\begin{array}{l}\mathrm{LDH}, \mathrm{U} / \mathrm{L} \\
\quad>225 \\
\quad \leq 225\end{array}$ & $135-225$ & $\begin{array}{l}185(148-230) \\
94(26.9) \\
256(73.1)\end{array}$ & $\begin{array}{l}223(|79-32|) \\
24(50.0) \\
24(50.0)\end{array}$ & $\begin{array}{l}182(145-220) \\
70(23.2) \\
232(76.8)\end{array}$ & $\begin{array}{l}<0.0001 \\
<0.0001\end{array}$ \\
\hline $\begin{array}{l}\text { Creatinine, } \mu \mathrm{mol} / \mathrm{L} \\
>106\end{array}$ & 54-106 & $\begin{array}{l}59.5(46.6-75.8) \\
14(4.0)\end{array}$ & $\begin{array}{l}59.6(47.7-71.7) \\
2(4.2)\end{array}$ & $\begin{array}{l}59.5(46 . \mid-76.0) \\
12(4.0)\end{array}$ & $\begin{array}{l}0.966 \\
0.949\end{array}$ \\
\hline
\end{tabular}

(Continued) 
Table 2 (Continued).

\begin{tabular}{|c|c|c|c|c|c|}
\hline & $\begin{array}{l}\text { Normal } \\
\text { Range }\end{array}$ & $\begin{array}{l}\text { All Patients } \\
(n=350)\end{array}$ & $\begin{array}{l}\text { Severe Patients } \\
(n=48)\end{array}$ & $\begin{array}{l}\text { Non-Severe } \\
\text { Patients } \\
(n=302)\end{array}$ & $P$ value \\
\hline $\begin{array}{l}\text { BUN, } \mathrm{mmol} / \mathrm{l} \\
\quad>8.2 \\
\quad \leq 8.2\end{array}$ & $2.86-8.2$ & $\begin{array}{l}4.3(3.4-5.4) \\
21(6.0) \\
329(94.0)\end{array}$ & $\begin{array}{l}5.1(4.2-8.2) \\
I I(22.9) \\
37(77.1)\end{array}$ & $\begin{array}{l}4.3(3.3-5.2) \\
10(3.3) \\
292(96.7)\end{array}$ & $\begin{array}{l}<0.0001 \\
<0.0001\end{array}$ \\
\hline $\begin{array}{l}\text { CRP, mg/L } \\
\quad>8\end{array}$ & $0-8$ & $\begin{array}{l}15.5(5.1-36.5) \\
235(67.1)\end{array}$ & $\begin{array}{l}39.1(18.1-75.9) \\
40(83.3)\end{array}$ & $\begin{array}{l}13.4(5.0-32.8) \\
195(64.6)\end{array}$ & $\begin{array}{l}<0.0001 \\
0.010\end{array}$ \\
\hline $\begin{array}{l}\mathrm{PCT}, \mathrm{ng} / \mathrm{mL} \\
\quad>0.05\end{array}$ & $0-0.05$ & $155(44.3)$ & $23(47.9)$ & $132(43.7)$ & 0.585 \\
\hline $\begin{array}{l}\text { Chest x-ray and CT findings } \\
\text { Normal } \\
\text { Unilateral pneumonia } \\
\text { Bilateral pneumonia }\end{array}$ & & $\begin{array}{l}37(10.6) \\
66(18.9) \\
247(70.6)\end{array}$ & $\begin{array}{l}0 \\
6(12.5) \\
42(87.5)\end{array}$ & $\begin{array}{l}37(12.3) \\
60(19.9) \\
205(67.9)\end{array}$ & $<0.0001$ \\
\hline Multiple mottling and ground-glass opacity & & $92(26.3)$ & $37(77.1)$ & $55(18.2)$ & $<0.0001$ \\
\hline
\end{tabular}

Notes: Data are presented as median, interquartile range or $n(\%)$.

Abbreviations: PT, prothrombin time; APTT, activated partial thromboplastin time; ALT, alanine aminotransferase; AST, aspartate aminotransferase; CK, creatine kinase; LDH, lactate dehydrogenase; BUN, blood urea nitrogen; CRP, C-creative protein; PCT, procalcitonin.

$19.5 \%, \quad P<0.0001)$, and intravenous immunoglobulin (IVIG) $(91.7 \%$ vs $17.5 \%, P<0.0001)$. Moxifloxacin was the most common antibiotic and was used in $70.8 \%$ of severe patients and $40.4 \%$ of non-severe patients. A total of $16.7 \%$ of COVID-19 patients used levofloxacin, and $14.6 \%$ received piperacillin tazobactam. Other antibiotics included cefoperazone sodium and sulbactam sodium, ceftriaxone, and several critical patients used meropenem, linezolid, or voriconazole. The median total dosage of methylprednisolone sodium succinate was $640 \mathrm{mg}$ (IQR, $360-960$ ) in severe patients, and $240 \mathrm{mg}$ (IQR, 160-400) in non-severe patients who showed progression $[P<0.0001]$. The median duration of methylprednisolone sodium succinate use was 8.5 days (IQR, 6.8-11.3) in the severe group

A
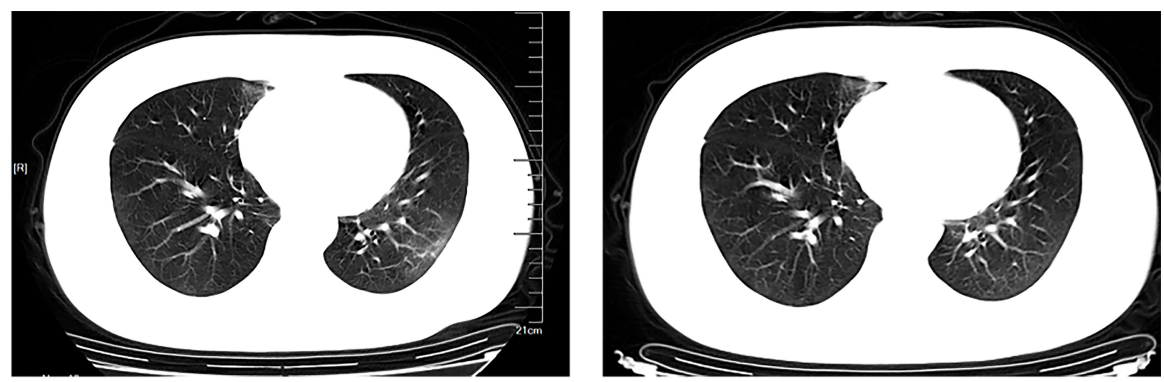

B
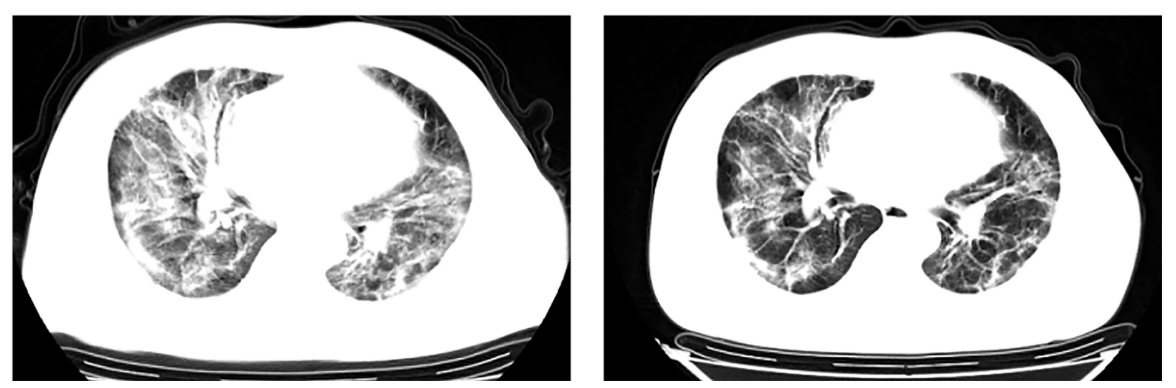

Figure 3 (A) CT images showed a ground-glass opacity (GGO) in the left pulmonary lobe of a 38-year-old female with mild symptoms on the first day of hospitalization, and follow-up images showed absorption on day 6. (B) CT images showed multiple mottling GGOs in a 63-year-old male with severe symptoms on the first day of admission, and follow-up images showed absorption on day 4. 
Table 3 Treatment and Outcomes of Patients Infected with SARS-CoV-2

\begin{tabular}{|c|c|c|c|c|}
\hline & $\begin{array}{l}\text { All Patients } \\
(n=350)\end{array}$ & $\begin{array}{l}\text { Severe } \\
\text { Patients } \\
(n=48)\end{array}$ & $\begin{array}{l}\text { Non-Severe } \\
\text { Patients } \\
(n=302)\end{array}$ & $P$ value \\
\hline Complications & $19(5.4)$ & $12(25.0)$ & $7(2.3)$ & $<0.0001$ \\
\hline ARDS & $4(1.1)$ & $4(8.3)$ & 0 & $<0.000 \mathrm{I}$ \\
\hline Acute cardiac injury & $2(0.6)$ & $2(4.2)$ & 0 & 0.018 \\
\hline Acute kidney injury & $4(1.1)$ & $4(8.3)$ & 0 & 0.0003 \\
\hline Acute hepatic injury & $2(0.6)$ & 0 & $2(0.7)$ & $>0.9999$ \\
\hline Sepsis & $4(1.1)$ & $4(8.3)$ & $0(0.0)$ & 0.0003 \\
\hline Allergic eruption & $7(2.0)$ & $2(4.2)$ & $5(1.7)$ & 0.247 \\
\hline Phlebothrombosis & $I(0.3)$ & $I(2 . I)$ & 0 & 0.137 \\
\hline Pneumothorax & $\mathrm{I}(0.3)$ & $I(2 . I)$ & 0 & 0.137 \\
\hline \multicolumn{5}{|l|}{ Treatment } \\
\hline Antiviral therapy & $326(93.1)$ & $46(95.8)$ & $280(92.7)$ & 0.553 \\
\hline Lopinavir and ritonavir & $5 I(14.6)$ & $6(12.5)$ & $45(14.9)$ & 0.661 \\
\hline Lopinavir and ritonavir plus recombinant human interferon- $\alpha 2 b$ & 157(44.9) & 19(39.6) & $138(45.7)$ & 0.429 \\
\hline Arbidol & $29(8.3)$ & $3(6.3)$ & $26(8.6)$ & 0.788 \\
\hline Arbidol plus recombinant human interferon- $\alpha 2 b$ & $40(11.4)$ & $8(16.7)$ & $32(10.6)$ & 0.219 \\
\hline Lopinavir and ritonavir plus arbidol & $49(14.0)$ & $10(20.8)$ & $39(12.9)$ & 0.142 \\
\hline Antibiotic therapy & $177(50.6)$ & $48(100.0)$ & $129(42.7)$ & $<0.0001$ \\
\hline Moxifloxacin & I56(44.5) & $34(70.8)$ & $122(40.4)$ & 0.0001 \\
\hline Levofloxacin & $25(7.2)$ & $8(16.7)$ & $17(5.6)$ & 0.012 \\
\hline Piperacillin tazobactam & $9(2.5)$ & $7(14.6)$ & $2(0.7)$ & $<0.0001$ \\
\hline Others $^{\dagger}$ & $\mathrm{II}(3.1)$ & $6(12.5)$ & $5(1.7)$ & 0.0013 \\
\hline Corticosteroid* & $105(30.0)$ & $46(95.8)$ & $59(19.5)$ & $<0.0001$ \\
\hline Total Dosage, $\mathrm{mg}$ & $360(200-600)$ & $640(360-960)$ & $240(160-400)$ & $<0.0001$ \\
\hline Duration, days & $4.0(4.0-9.0)$ & $8.5(6.8-11.3)$ & $5.0(4.0-6.0)$ & $<0.0001$ \\
\hline Intravenous immunoglobulin therapy & $97(27.7)$ & $44(91.7)$ & $53(17.5)$ & $<0.000$ I \\
\hline Total Dosage, $g$ & $60(45-90)$ & $80(60-140)$ & $50(10-60)$ & $<0.0001$ \\
\hline Duration, days & $6.0(5.0-9.0)$ & $8.0(6.0-11.5)$ & $5.0(3.0-6.0)$ & $<0.0001$ \\
\hline Oxygen support & $263(75.1)$ & $48(100.0)$ & $215(7 \mid .2)$ & $<0.0001$ \\
\hline Nasal cannula & $229(65.4)$ & 14(29.2) & $215(7 \mid .2)$ & $<0.0001$ \\
\hline High-flow nasal cannula & $20(5.7)$ & $20(41.7)$ & 0 & $<0.0001$ \\
\hline Non-invasive ventilation & $14(4.0)$ & 14(29.2) & 0 & $<0.0001$ \\
\hline Invasive mechanical ventilation & $6(1.7)$ & $6(12.5)$ & 0 & $<0.0001$ \\
\hline ECMO & $5(1.4)$ & $5(10.4)$ & 0 & $<0.0001$ \\
\hline \multicolumn{5}{|l|}{ Prognosis } \\
\hline Discharge & $348(99.4)$ & $46(95.8)$ & $302(100.0)$ & 0.018 \\
\hline Death & $2(0.57)$ & $2(4.2)$ & 0 & \\
\hline Onset of symptoms to negative RT-PCR results, days & $16(12-21)$ & $18(13.5-22.5)$ & $16(12-20)$ & 0.065 \\
\hline Onset of admission to Discharge, days & $\mid 4(||-2 \mid)$ & $15(11-25)$ & $\mid 4(||-20)$ & 0.536 \\
\hline
\end{tabular}

Notes: Data are presented as median, interquartile range or $\mathrm{n}(\%)$. ${ }^{\dagger}$ Other antibiotics included cefoperazone sodium and sulbactam sodium, ceftriaxone, and several critical patients used meropenem, linezolid, or voriconazole. *Corticosteroid used in our cohort was methylprednisolone sodium succinate.

Abbreviations: ARDS, acute respiratory distress syndrome; ECMO, extracorporeal membrane oxygenation.

and 5.0 days (IQR, 4.0-6.0) in the non-severe group for those who showed progression $[P<0.0001]$. The median total dosage of IVIG was $80 \mathrm{~g}$ (IQR, 60-140) in severe patients, and $50 \mathrm{~g}$ (IQR, 10-60) in non-severe patients with progression $[P<0.0001]$. The median duration of IVIG use were 8.0 days (IQR, 6.0-11.5) in the severe group and 5.0 days (IQR, 3.0-6.0) in the non-severe group for those with progression $[P<0.0001]$. One hundred percent of severe patients received oxygen support, and the corresponding proportion was $71.2 \%$ in non-severe patients $[P<0.0001]$. No non-severe patients received support from a mechanical ventilator. The time until a negative RT-PCR result was not 
statistically significant between the two groups (median 18 days, IQR, 13.5-22.5 vs median 16 days, IQR, 12-20) $[P=0.065]$. The duration from admission to discharge was also no statistically significant between the two groups (median 15 days, IQR, 11-25 vs median 14 days, IQR, 11-20) $[P=0.536]$. As of March 15, 2020, all patients have been discharged. Two deaths occurred in the severe group. One severe male patient with a medical history of coronary heart disease and chronic obstructive pulmonary disease (COPD), aged 64, improved and got successfully endotracheal extubation initially, but several days later, he deteriorated suddenly with cardiac respiratory arrest and died. Laboratory findings suggested acute myocardial injury. The other death occurred in a 58-year-old male with chronic hepatitis B and COPD, who died of respiratory failure.

\section{Risk Factors for Severe Illness}

As some patients were classified as severe cases at admission, we chose epidemiological factors to explore the risk factors associated with severe COVID-19. In the univariable analysis, the odds ratio (OR) of severe COVID-19 was higher for patients who were aged 60 years and older (OR 5.04, 95\% CI 2.62-9.58, $P<0.0001$ ), had a history of exposure to Wuhan (OR 1.25, 95\% CI $0.67-2.31, P=0.048$ ), had a smoking history (OR 4.14, 95\% CI 1.85-9.24, $P=0.001$ ), or had chronic disease (OR 4.3, 95\% CI 2.2-8.1, $P<0.0001$ ) including hypertension (OR 3.8, 95\% CI 1.89-7.62, $P<0.0001$ ), diabetes (OR $3.85,95 \%$ CI 1.61-9.2, $P=0.002$ ), cardiovascular disease (OR 8.4, 95\% CI 2.9-24.4, $P<0.0001$ ), or chronic liver disease (OR 3.77, 95\% CI 1.21-11.78, $P=0.022$ ). Further multivariable logistic regression models showed that an age above 60 years old was associated with increased odds for severe disease (OR 4.14, 95\% CI 1.73-9.84, $P=0.001$ ). Family cluster infection was associated with reduced odds of severe illness (OR 0.46, 95\% CI 0.22-0.97, $P=0.042$ ) (Table 4).

\section{Discussion}

In this retrospective, multicenter study, a total of 350 patients were enrolled. Forty-eight COVID-19 patients in Hunan Province were considered severe cases, including 15 critically severe patients. An age above 60 years old was a potential risk factor for severe disease. The severe group had more patients with a history of smoking and chronic medical illness, especially hypertension and diabetes. A fever above 38 degrees and dyspnea were ominous in the severe ill on admission. There was no obvious difference regarding the median time from illness onset to hospital admission between severe and non-severe patients, and this duration was 5 days. Moreover, the median time from symptom onset to a negative PCR result was similar in both groups.

In terms of laboratory tests, severe patients had more abnormal laboratory results than non-severe cases, including lower lymphocyte counts, lower albumin, higher globulin, higher AST, higher myocardial zymogram, higher BUN, more prolonged APTT, and more thrombopenia. Particularly, high levels of CRP, a D-dimer $>1$, and a granulocyte/lymphocyte ratio $>3$ were good tools to distinguish between severe and non-severe patients according to our study. These laboratory findings were in accordance with results reported from other studies, such as indexes related to the immune response including lower lymphocytes, higher CRP, and higher granulocyte/lymphocyte ratio were frequent in severe COVID-19 patients, ${ }^{13}$ especially the change of CRP are very sensitive to disease progression. ${ }^{14}$ Notably, we first found the ratio of granulocyte/lymphocyte beyond 3 is an ideal indicator to distinguish severe patients. Other parameters such as abnormal AST, myocardial zymogram, BUN, APTT, and D-dimer, in the combination of other studies, have shown common multi-organ functional disturbance in severe patients. ${ }^{15-17}$ The CT features were different, as we showed that severe patients presented with more bilateral pneumonia or multiple mottling and ground-glass opacities (GGOs), which was consistent with a previous study that showed that CT was important in determining of the severity of the disease. ${ }^{18}$

In this study, the median age of the severe patients was 61 years old, while the median age was 42 years old in non-severe group. Logistic regression further confirmed that an age above 60 years old was a potential risk factor for the severe disease. A recent study from Wuhan also confirmed that older age was a risk factor for death due to SARS-CoV-2 infection. ${ }^{19}$ The likelihood of poor outcomes in aged patients may be attributed to T-cellular immune deficiency and the degeneration of organ function with aging. ${ }^{20,21}$ Special attention and careful management are necessary for patients aged above 60 years old and for those with chronic medical illness, as our study also showed that more severe patients had comorbidities, including hypertension and diabetes, which was consistent with the study from Guan et al. ${ }^{22}$

There were fewer severe patients, including critical patients, in Hunan $(13.7 \%)$ than in Wuhan. Logistic regression showed that patients infected by family clusters had reduced odds of progressing into severe COVID-19, 
Table 4 Risk Factors Associated with Severe COVID-19

\begin{tabular}{|c|c|c|c|c|}
\hline & $\begin{array}{l}\text { Univariable OR } \\
(95 \% \mathrm{Cl})\end{array}$ & $\mathrm{p}$ value & $\begin{array}{l}\text { Multivariable } \\
\text { OR }(95 \% \mathrm{Cl})\end{array}$ & p value \\
\hline \multicolumn{5}{|l|}{ Age, years } \\
\hline$\geq 60$ & $5.04(2.62-9.58)$ & $<0.000$ I & $4.14(1.73-9.84)$ & 0.001 \\
\hline Male sex & $1.67(0.91-3.16)$ & 0.095 & $2.35(0.85-6.5 I)$ & 0.100 \\
\hline Wuhan exposure & $1.25(0.67-2.31)$ & 0.048 & I.19(0.58-2.46) & 0.627 \\
\hline Family cluster case & $0.69(0.37-1.29)$ & 0.245 & $0.46(0.22-0.97)$ & 0.042 \\
\hline Smoking & $4.14(1.85-9.24)$ & 0.001 & $2.35(0.85-6.5 I)$ & 0.100 \\
\hline Comorbidity & $4.3(2.2-8.1)$ & $<0.000$ I & $1.38(0.49-3.83)$ & 0.539 \\
\hline Hypertension & $3.8(1.89-7.62)$ & $<0.000$ I & $0.92(0.29-2.91)$ & \\
\hline Diabetes & $3.85(I .6 I-9.2)$ & 0.002 & $2.01(0.63-6.83)$ & 0.233 \\
\hline Cardiovascular disease & $8.4(2.9-24.4)$ & $<0.0001$ & $\mathrm{I} .8 \mathrm{I}(0.42-7.84)$ & 0.424 \\
\hline Chronic kidney disease & $1.82(0.36-9.06)$ & $0.46 \mathrm{I}$ & $0.81(0.12-5.55)$ & 0.827 \\
\hline Chronic liver disease & $3.77(1.21-11.78)$ & 0.022 & $2.30(0.48-10.90)$ & 0.297 \\
\hline Cerebral infarction & $3.95(0.91-17.01)$ & 0.066 & I.27(0.22-7.3I) & 0.786 \\
\hline
\end{tabular}

which indicates that patients of secondary or tertiary generations have a lower incidence of severe illness. These findings suggest that the incidence of severe disease would be reduced with transmission. This may be attributed to timely quarantine and effective management based on different clinical classifications. In Hunan, all infected were quarantined and managed in designated hospitals, like Fangcang shelter hospitals ${ }^{23}$ in Wuhan. Asymptomatic carriers and non-severe patients received examinations regularly; when they progressed, interventions were adopted in a timely and early manner. Severe patients were admitted to the ICU immediately to receive better observation and treatment.

At present, no specific drug has been recommended for COVID-19, although some clinical trials have shown that remdesivir, ${ }^{24}$ favipiravir, ${ }^{25}$ and combined therapy including hydroxychloroquine and azithromycin ${ }^{26}$ may be effective; due to the limited samples and trial designs, further confirmation is needed. Systemic therapy is recommended by the National Health Commission of China and WHO, ${ }^{12,27}$ but details may vary in different areas. Most severe patients were discharged, and only two deaths occurred in our cohort, so we summarized the therapeutic features as follows: First, other than asymptomatic carriers and mild patients, most patients received antivirals such as lopinavir and ritonavir plus recombinant human interferon$\alpha 2 b$ when admitted. A study ${ }^{28}$ also revealed that the early use of antiviral agents, such as lopinavir and ritonavir could improve the prognosis of severe patients. Before powerful evidence of specific antivirals is available, lopinavir and ritonavir plus recombinant human interferon- $\alpha 2 b$ could still be used.

Second, severe patients and non-severe patients with progressive manifestations including persistent high fever and dyspnea, rapid progressive CT images, and worsening lymphopenia, could receive IVIG plus corticosteroids, as the progression of the SARS-CoV-2 infection is associated with reduced immunity and proinflammatory cytokine storms. ${ }^{29,30}$ In our cohort, IVIG was used at $10 \mathrm{~g} /$ day in severe patients, $20 \mathrm{~g}$ /day in critical patients, and less than $10 \mathrm{~g} /$ day in non-severe patients with progression. The duration of use was dependent on the improvement of the disease, and was usually approximately 8.0 days in severe patients and 5.0 days in the non-severe group. Lin et $\mathrm{al}^{29}$ recommended IVIG for $0.3-0.5 \mathrm{~kg}$ /day and 5 days, but the usage of IVIG still needs exploration. The use of corticosteroids in COVID-19 remains controversial, as some researchers think corticosteroid may increase mortality, secondary infection and impair viral clearance. ${ }^{31}$ A recent study showed that the proper use of corticosteroids would not delay SARS-CoV-2 clearance. ${ }^{32}$ From the perspective of front-line physicians in China, low-tomoderate doses of corticosteroids were effective and few serious side effects were observed in COVID-19 patients. ${ }^{33}$ Before the use of corticosteroids, contraindications including serious secondary infection should be excluded, and the dosage may vary with different recommendations. The guidelines of the National Health Commission of China suggest methylprednisolone $1-2 \mathrm{mg} / \mathrm{kg} /$ day for $3-5$ days in severe patients, ${ }^{12}$ while 
Shang et $\mathrm{al}^{33}$ believed that $\leq 0.5-1 \mathrm{mg} / \mathrm{kg} /$ day for $\leq 7$ days could be used. In our cohort, 80-160 mg/day of methylprednisolone sodium succinate was used in severe patients, $40-80 \mathrm{mg}$ /day was used in non-severe patients with progression, and the median duration of use was approximately 8.5 days and 5.0 days, respectively. We usually used corticosteroids within a short time and reduced the dosage gradually when the patients improved. However, in some patients with slow improvement or reaggravation during the dosage reduction, the duration was prolonged; although few short-term side effects were observed, longterm complications need further investigation. Considering current controversy, well-designed trials are still needed to provide more information about corticosteroid use in COVID-19.

Third, for patients with increased PCT, yellow sputum, or severe disease, antibiotics were used to prevent or treat secondary infection, and the most frequent type was moxifloxacin. If the patients' condition deteriorated, especially some critically severe patients, other antibiotics, such as piperacillin tazobactam, meropenem, or linezolid were added. Few patients used voriconazole as a therapy for aspergillus infection. We were cautious about using invasive mechanical ventilation except for severe patients whose condition progressed rapidly or those who had uncontrolled hypoxemia after strategic interventions of oxygen support through high-flow nasal cannula or noninvasive mechanical ventilation. Last but not least, we suggest checking the laboratory results and CT images every three days for severe patients and non-severe patients with progressive manifestations. When the patient is improved and stable, the time interval for the reexaminations could be extended to five days.

This study has several limitations. First, as we included patients classified as severe at admission, we were unable to estimate risk factors such as laboratory results to predict severe COVID-19. Moreover, the observation time of severe COVID-19 patients was limited, and follow-up is necessary to observe the future prognosis of these severe patients, such as the long-term side effects of corticosteroids, impact on pulmonary function or possibility of lung fibrosis.

\section{Conclusion}

In conclusion, the rate of severe cases and mortality rate of COVID-19 in Hunan were relatively lower than those associated with the original COVID-19 outbreak in Wuhan. Patients aged above 60 years old were more susceptible to severe COVID-19, and this factor does not change over different generations of SARS-CoV-2. CT scans and multiple laboratory examinations are important in identifying severe cases. In addition to antivirals and oxygen support, timely interventions including corticosteroid, intravenous IVIG, and antibiotics are very important to improve the prognosis of severe COVID-19 patients.

\section{Ethics Statement}

This study was approved by the Medical Ethical Committees of the Public Health Clinic Center of Changsha City, Central Hospital of Zhuzhou City, First People's Hospital of Huaihua City, and the Second Xiangya Hospital of Central South University (Approved Number. KL-2,020,020), and the requirement for informed consent was waived based on the CIOMS guidelines.

\section{Funding}

This study was funded by the National Natural Science Foundation of China (No. 81370164 and No. 81670062), the National Natural Science Foundation of Hunan Province (No. 2015JJ4087 and No. 2020JJ8070), the National key clinical specialist construction Programs of China, the Innovative Major Emergent Project against the Outbreak of New Coronavirus Pneumonia in Hunan Province (No. 2020SK3014), the Fundamental Research Funds for the Central Universities of Central South University (No. 2020zzts880), and the China Scholarship Council (No. 201906370230). These first authors contributed equally to this work: Ting Guo, Qinxue Shen.

\section{Disclosure}

The authors report no conflicts of interest in this work.

\section{References}

1. Na Z, Dingyu Z, Wenling W, et al. A novel coronavirus from patients with pneumonia in China, 2019. N Engl J Med. 2020;382(8):727-733. doi:10.1056/NEJMoa2001017

2. National Health Commission of the People's Republic China. The latest situation of new coronavirus pneumonia. Available from: http://www. nhc.gov.cn/xcs/yqfkdt/202004/9631d3d14b514055ac74b9b4d1367904. shtml.

3. World Health Organization. WHO coronavirus disease (COVID-19) dashboard. Available from: https://covid19.who.int/.

4. World Health Organization. General's opening remarks at the mission briefing on COVID-19-12; 2020. Available from: https://www.who.int/ $\mathrm{dg} /$ speeches/detail/who-director-general-s-opening-remarks-at-themission-briefing-on-covid-19-12-march-2020.

5. Chaolin H, Yeming W, Xingwang L, et al. Clinical features of patients infected with 2019 novel coronavirus in Wuhan, China. Lancet. 2020;395(10223):497-506. doi:10.1016/S0140-6736(20)30183-5 
6. Dawei W, Bo H, Chang $\mathrm{H}$, et al. Clinical characteristics of 138 hospitalized patients with 2019 novel coronavirus-infected pneumonia in Wuhan, China. JAMA. 2020; 201585.

7. Nanshan C, Min Z, Xuan D, et al. Epidemiological and clinical characteristics of 99 cases of 2019 novel coronavirus pneumonia in Wuhan, China: a descriptive study. Lancet. 2020;395 (10223):507-513. doi:10.1016/S0140-6736(20)30211-7

8. Jinjin Z, Xiang D, Yiyuan C, et al. Clinical characteristics of 140 patients infected by SARS-CoV-2 in Wuhan, China. Allergy. 2020.

9. Sijia T, Nan H, Jing L, et al. Characteristics of COVID-19 infection in Beijing. $J$ Infect. 2020;80(4):401-406.

10. Xiao-Wei X, Xiao-Xin W, Xian-Gao J, et al. Clinical findings in a group of patients infected with the 2019 novel coronavirus (SARS-Cov-2) outside of Wuhan, China: retrospective case series. BMJ. 2020;368:m606.

11. Jian W, Jun L, Xinguo Z, et al. Clinical characteristics of imported cases of COVID-19 in Jiangsu Province: a multicenter descriptive study. Clin Infect Dis. 2020; ciaa199.

12. Chinese National Health Committee. The diagnostic and treatment guideline for novel coronavirus pneumonia (version six). Available from: http://www.nhc.gov.cn/yzygj/s7653 p/202002/ 8334a8326dd94d329df351d7da8aefc2.shtml.

13. Xiaolei L, Yang L, Junming L, et al. Immune characteristics distinguish patients with severe disease associated with SARS-CoV-2. Immunol Res. 2020;1-7.

14. Chaochao T, Ying H, Fengxia S, et al. C-reactive protein correlates with computed tomographic findings and predicts severe COVID-19 early. J Med Virol. 2020;92(7):856-862. doi:10.1002/jmv.25871

15. Aliye B, Hurrem B, Serpil E, et al. Clinical and laboratory features of COVID-19: predictors of severe prognosis. Int Immunopharmacol. 2020;88:106950.

16. Edith S, Guillaume P, Violeta S, et al. Covid-19: contribution of clinical characteristics and laboratory features for early detection of patients with high risk of severe evolution. Acta Clin Belg. 2020;1-7.

17. Aining Z, Yan L, Yi Z, et al. Meta-analysis of coagulation parameters associated with disease severity and poor prognosis of COVID-19. Int J Infect Dis. 2020.

18. Wei Z, Zheng Z, Xingzhi X, et al. Relation between chest CT findings and clinical conditions of coronavirus disease (COVID-19) pneumonia: a multicenter study. AJR Am J Roentgenol. 2020;1-6.

19. Fei Z, Ting Y, Ronghui D, et al. Clinical course and risk factors for mortality of adult inpatients with COVID-19 in Wuhan, China: a retrospective cohort study. Lancet. 2020;395(10229):1054-1062.

20. Ling Q, Xie J, Zhifeng Q, et al. Aging of immune system: immune signature from peripheral blood lymphocyte subsets in 1068 healthy adults. Aging (Albany NY). 2016;8(5):848-859.
21. Liu K, Chen Y, Lin R, Han K. Clinical features of COVID-19 in elderly patients: a comparison with young and middle-aged patients. $J$ Infect. 2020.

22. Guan WJ, Liang WH, Zhao Y, et al. Comorbidity and its impact on 1590 patients with Covid-19 in China: a nationwide analysis. Eur Respir J. 2020;2000547.

23. Simiao C, Zongjiu Z, Juntao Y, et al. Fangcang shelter hospitals: a novel concept for responding to public health emergencies. Lancet. 2020;S0140-6736(20)30744-3.

24. Grein J, Ohmagari N, Shin D, et al. Compassionate use of remdesivir for patients with severe covid-19. $N$ Engl J Med. 2020;382 (24):2327-2336. doi:10.1056/NEJMoa2007016

25. Chang $\mathrm{C}$, Jianying $\mathrm{H}$, Zhenshun $\mathrm{C}$, et al. Favipiravir versus arbidol for COVID-19: a randomized clinical trial. medRxiv. 2020.

26. Gautret P, Lagier JC, Parola P, et al. Hydroxychloroquine and azithromycin as a treatment of COVID-19: results of an open-label non-randomized clinical trial. Int J Antimicrob Agents. 2020;56 (1):105949. doi:10.1016/j.ijantimicag.2020.105949

27. World Health Organization. Clinical management of severe acute respiratory infection when novel coronavirus $(\mathrm{nCoV})$ infection is suspected: interim guidance; 2020. Available from: https:/www.who.int/ publications-detail/clinical-management-of-severe-acute-respiratoryinfection-whennovel-coronavirus-(ncov)-infection-is-suspected.

28. Wu J, Li W, Shi X, et al. Early antiviral treatment contributes to alleviate the severity and improve the prognosis of patients with novel coronavirus disease (COVID-19). J Intern Med. 2020;288 (1):128-138. doi:10.1111/joim.13063

29. Lin L, Lianfeng L, Wei C, Taisheng L. Hypothesis for potential pathogenesis of SARS-CoV-2 infection-a review of immune changes in patients with viral pneumonia. Emerg Microbes Infect. 2020;9 (1):727-732. doi:10.1080/22221751.2020.1746199

30. Jing L, Sumeng L, Jia L, et al. Longitudinal characteristics of lymphocyte responses and cytokine profiles in the peripheral blood of SARS-CoV-2 infected patients. medRxiv. 2020.

31. Russell CD, Millar JE, Baillie JK. Clinical evidence does not support corticosteroid treatment for $2019-\mathrm{nCoV}$ lung injury. Lancet. 2020;395(10223):473-475. doi:10.1016/S0140-6736(20)30317-2

32. Xiaowei F, Qing M, Tianjun Y, et al. Low-dose corticosteroid therapy does not delay viral clearance in patients with COVID-1. J Infect. 2020.

33. Shang L, Jianping Z, Yi H, Ronghui D, Bin C. On the use of corticosteroids for 2019-nCoV pneumonia. Lancet. 2020;395 (10225):683-684. doi:10.1016/S0140-6736(20)30361-5
Infection and Drug Resistance

\section{Publish your work in this journal}

Infection and Drug Resistance is an international, peer-reviewed openaccess journal that focuses on the optimal treatment of infection (bacterial, fungal and viral) and the development and institution of preventive strategies to minimize the development and spread of resistance. The journal is specifically concerned with the epidemiology of antibiotic resistance and the mechanisms of resistance development and diffusion in both hospitals and the community. The manuscript management system is completely online and includes a very quick and fair peerreview system, which is all easy to use. Visit http://www.dovepress.com/ testimonials.php to read real quotes from published authors. 